\title{
RELATIONSHIP BETWEEN PYOCINS AND A BACTERIOPHAGE IN PSEUDOMONAS AERUGINOSA ${ }^{1}$
}

\author{
SEIGA ITO ${ }^{2}$ AND MAKOTO KAGEYAMA \\ Department of Biophysics and Biochemistry, Faculty of Science, University \\ of Tokyo, Hongo, Tokyo
}

(Received January 14, 1970)

\begin{abstract}
A bacteriophage related to R-type pyocins was isolated from a lysogenic strain of Pseudomonas aeruginosa and named PS3. It was composed of a head and a contractile tail which resembled R-type pyocins. The character of the phage was studied comparing with R-type pyocins.

The activity of this phage was neutralized by anti- $\mathrm{R}$ or $\mathrm{R} 2$ serum and the antiserum against PS3 was also active in inhibiting pyocins R and R2. However, the anti- $R$ sheath serum, while showing a strong neutralizing activity against pyocin $\mathrm{R}$ or $\mathrm{R} 2$, was almost ineffective against the phage PS3.

The phage and the pyocins were further related in some way in the receptor specificity. Mutants resistant to one of the killer particles were isolated and their behavior to other agents were studied. From this result, a working hypothesis was presented with regard to the structure of the receptor for the phage and pyocins.
\end{abstract}

Bacteriocins are defined as a group of proteins which are produced by certain bacteria and have a killing action on other strains of the same or closely related family. Since the discovery of bacteriocins, they have often been compared with bacteriophages (1-3). Both agents are adsorbed by specific receptors of the cell wall and kill the sensitive bacteria. In some cases, receptors are common for certain bacteriocins and phages; colicin $\mathrm{K}$ and phage $\mathrm{T} 6$ or colicin $\mathrm{E}$ and phage BF23. Both lysogenic bacteria and bacteriocinogenic bacteria are immune against the agents they produce. Lysogeny and bacteriocinogeny are both potential characters and production of the agents is often inducible by some kind of treatments like ultraviolet irradiation and is a lethal process for either bacterium. However, there is an essential difference between bacteriocins and phages, as the latter is selfreproducible in the sensitive bacteria while the former is not.

1 A part of this study was presented at the 40th Annual Meeting of Japanese Biochemical Society, Tokyo, Oct. 28, 1968.

2 Present Address; Tokyo Research Laboratory, Kyowa Hakko Kogyo Co., Ltd., Machida, Tokyo. 
We have been studying pyocins, the bacteriocins of Pseudomonas aeruginos $a$, and found that some of them had a structure very similar to some phage tails and we called them R-type pyocins (4). Several kinds of killer particles with a phage-like structure have recently been reported among other bacteria $(5-8)$. These findings may provide some suggestion about the origin of bacteriocins as well as bacteriophages.

Recently, Homma and his colleagues found several Pseudomonas phages which showed immunological cross reactions with a pyocin $(9,10)$. Such phages should be very useful in studying the origin, mode of action and production, and genetics of the pyocins. We also succeeded in finding a phage, named PS3, which was immunologically related to R-type pyocins, R, R2, R3, and R4, which we found among several strains of Pseudomonas (4). In this paper, we will describe the nature of the phage and its relation to R-type pyocins from the standpoints of structure, immunology, and the receptor specificity.

\section{MATERIALS AND METHODS}

Bacterial strains. For the preparation of pyocins, Pseudomonas aeruginosa strain P15 (for pyocin R), M11 (pyocin R2), M2008 (pyocin R3), and P28 (pyocin R4) were employed.

As indicator strains for pyocins and a phage, P14 and P15-16 were used. P15-16 is a strain derived from P15 by treatment with N-methyl-N'nitro-N-nitrosoguanidine and has the following character: $\operatorname{trp}^{-}$, $\operatorname{str}^{\mathrm{r}}$, pyocin $\mathrm{R}^{-}$, pyocin $\mathrm{R}^{\mathrm{s}}$. $\mathrm{M} 78$ was also employed as a phage indicator.

Strains prefixed with $\mathrm{P}$ are the derivatives of Dr. J.Y. Homma's strains and those prefixed with $M$ are derived from Dr. B.W. Holloway's strains. The detailed characters of above-mentioned strains were listed in the preceding paper (4).

The phage, named PS3 in this paper, was isolated from a lysogenic strain P1 kindly donated by Dr. J.Y. Homma. When it is necessary to specify on which strain the phage was propagated, the phage preparation will be denoted by appending the name of the strain in parentheses, thus PS3 (P15-16).

Culture and assay conditions. Culture media and the preparation of pyocins (R, R2, R3 and R4) were the same as described in the preceding paper (4). Preparation of the phage sample was made as follows: A culture of sensitive strain was infected with the phage at the logarithmic phase of growth. The multiplicity of infection was around 0.2. A clear lysate was obtained after $3-4 \mathrm{hr}$ of cultivation with a titer of about $1 \times 10^{10} \mathrm{PFU}$ (plaque forming unit) per $\mathrm{ml}$. The phage titration method and general phage techniques were essentially the same as described by ADAMS (11). The pyocin activity was assayed by the method described previously (12).

Preparation of antisera against pyocins and the phage. Anti-pyocin $\mathrm{R}$ or pyocin R2 serum was prepared as follows: About $1 \mathrm{mg}$ of purified pyocin 
prepared by KAGEYAMA's method (13) was mixed with the Freund's complete adjuvant and injected into rabbits. The second injection was carried out after 1 month and the rabbits were sacrificed 1 week later. Anti-pyocin $\mathrm{R}$ sheath serum was prepared by Miss $\mathrm{C}$. Yui in our laboratory. Purified pyocin $\mathrm{R}$ was disintegrated into the sheath and other components by treatment with dilute alkali and the sheath was isolated by the sucrose density gradient centrifugation (14). The sheath fraction was dissolved in $0.1 \% \mathrm{NaCl}$ and was used as an antigen.

When the antiserum against the phage PS3 was prepared, the phage was propagated on the strain P15-16 to avoid the contamination of pyocin, as this strain was known not to produce any pyocin. The phage was purified by repeating low- and high-speed centrifugations $(20,000 \times g$, $45 \mathrm{~min})$. The concentrated phage solution was used as an antigen and the antiserum was prepared in the same way as described above.

Assay of antiserum activity neutralizing the phage or pyocins. All the dilution was performed with the nutrient broth. A phage solution diluted to $3 \times 10^{8} \mathrm{PFU} / \mathrm{ml}$ and $0.9 \mathrm{ml}$ portions of serially diluted antiserum solutions were preincubated at $37^{\circ}$. A volume of $0.1 \mathrm{ml}$ of the phage solution was added to each diluted antiserum solution. The mixtures were kept at $37^{\circ}$ for $5 \mathrm{~min}$ and then $0.1 \mathrm{ml}$ portions of serially diluted samples were plated with an indicator strain P15-16 in a soft agar solution. The plaques were counted after overnight incubation at $37^{\circ}$. The velocity of neutralization by the serum can be expressed by $\mathrm{K}$ value calculated according to the formula: $\mathrm{K}=2.3 \mathrm{D} / t \cdot \log \mathrm{P}_{0} / \mathrm{P}$, where $\mathrm{D}$ is the final dilution of serum in the phage-serum mixture, $\mathrm{P}_{0}$ the phage titer at 0 time, and $\mathrm{P}$ the phage titer at $t$ min. $\mathrm{K}$ values were calculated from the experiments where $90 \sim 99 \%$ of the phage activity was lost in $5 \mathrm{~min}$.

$\mathrm{K}$ values of the neutralization of pyocins were estimated as follows: A purified pyocin solution and serially diluted serum solutions were preincubated at $37^{\circ}$. The pyocin solution $(0.5 \mathrm{ml})$ and a serum solution $(0.5 \mathrm{ml})$ were mixed at $37^{\circ}$. After $5 \mathrm{~min}$, the mixture was diluted to $1: 100$ to stop the reaction, and the remaining pyocin activity was assayed after binary dilutions (12). Strain P14 was used for the assay of pyocin R and P15-16 for pyocin R2. An inhibition of growth of indicator strain was observed by the control sample (no serum) up to $2^{4}$ dilution (i.e. 1,600 units $/ \mathrm{ml}$ in the original mixture). The dilution of serum required to reduce the pyocin activity to $1 / 16$ was adopted to calculate the $\mathrm{K}$ value. Thus, $\mathrm{K}=2.3 \mathrm{D} / t \cdot \log \mathrm{P}_{0} / \mathrm{P}=2.3 \mathrm{D} / 5 \cdot \log$ 16 , where $\mathrm{D}$ is the final dilution of serum in the pyocin-serum mixture. As the assay of pyocin activity is not very accurate, $\mathrm{K}$ values obtained for pyocins are not so reliable as those for the phage.

Isolation of strains resistant to the phage or pyocins. A $0.1-\mathrm{ml}$ portion of an actively growing culture of P15-16 was spread over a nutrient agar plate and a purified pyocin or partially purified phage solution was added on it. After an overnight incubation, resistant colonies that appeared were 
picked up, purified by restreaking, and tested again to confirm their resistance to the phage or the pyocin employed. In the case of phage resistance the mutants were tested to ascertain that they were not lysogenized by the phage.

\section{RESULTS}

Isolation of phage PS3. An actively growing culture of strain P1 in the nutrient broth was treated with Mitomycin-C $(1.5 \mu \mathrm{g} / \mathrm{ml})$. The culture lysed after $2 \mathrm{hr}$. The resulting clear lysate was examined for phages with various Pseudomonas strains. Small turbid plaques (about $0.5 \mathrm{~mm}$ in diameter) appeared on strain P15 at about $2 \times 10^{6} / \mathrm{ml}$ and minute clear plaques (less than $0.5 \mathrm{~mm}$ ) were found on strain P14 at about $2 \times 10^{4} / \mathrm{ml}$ of the lysate. The number of plaques of the latter type was very much decreased by the addition of $0.1 \mathrm{ml}$ of antipyocin $\mathrm{R}$ serum to the soft agar for phage assay. This phage was purified by a single plaque isolation and named PS3. A study of the host range of this phage revealed a typical restriction and modification phenomenon, and this point will be described later. As this phage forms slightly bigger and more distinct plaques on P15-16 than on P14, a phage preparation PS3 (P15-16) was mainly employed in this study.

Some characteristics of the phage PS3. The growth characteristic of PS3 was examined in the nutrient broth at $37^{\circ}$ using P15-16 as a host strain. The latent period was found to be $60 \mathrm{~min}$ and the burst size was 30-40. The phage never lysogenized in either strain P14 or P15-16.

The plaque-forming activity of the phage solution was not stable in the presence of $\mathrm{CHCl}_{3}$. The titer of the phage lysate stored at $4^{\circ}$ with a drop of $\mathrm{CHCl}_{3}$ decreased gradually with a half-life time of about 5 days. The Millipore-filtered suspension seemed less unstable.

Electron microscopic observation. A partially purified preparation of PS3 was examined by an electron microscope by negative staining with phosphotungstate. As shown in Fig. 1, the phage is composed of a polyhedral head and a tail with a contractile sheath and a core. The dimension of the phage is schematically illustrated in Fig. 2 comparing to that of pyocin R4. The tail of the phage highly resembles $\mathrm{R}$ type pyocins reported in the preceding paper (4), except that the size of the tail is a little longer than that of pyocins. The electron microscopy of the phage preparation often disclosed particles with empty heads and contracted sheaths (Fig. 1, left). It is not yet clear whether the phage has any other structure like a base plate and tail fibers.

Host-controlled restriction and modification of the phage. During the survey of the host range of the phage PS3, a considerable difference in the efficiency of plating was found with several strains. It has been known that the host range of some phages is modified phenotypically after growth in a new host, while a phage which does not carry a modification pattern com- 


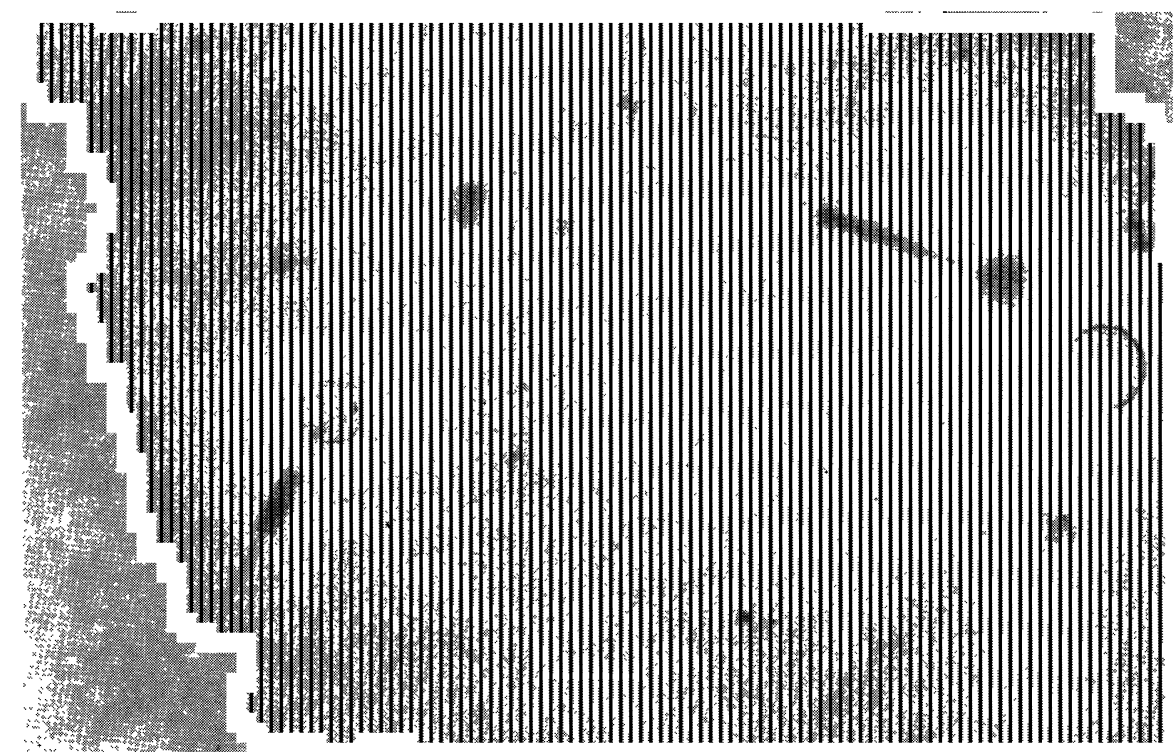

Fig. 1. Electron micrograph of phage PS3.

The scale in the picture indicates $1,000 \AA$.

Phage PS3

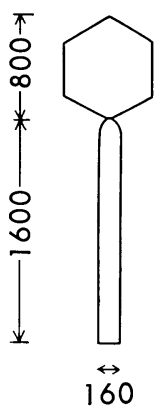

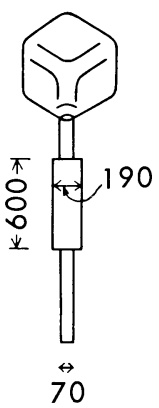

Shrunk
Pyocin R4

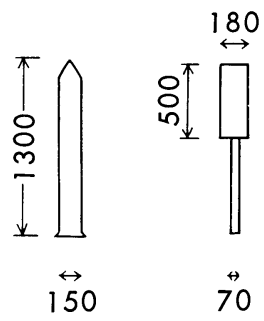

Intact Shrunk

Fig. 2. Morphological comparison of phage PS3 and R-type pyocin.

Dimensions are shown in $\AA$. Finer structures are omitted. It has been shown that pyocin $\mathrm{R}$ has a base plate and tail fibers (17). This point is not yet clear in this phage. 
Table 1. Host-controlled restriction of phage PS3.

\begin{tabular}{c|c|c|c}
\hline \multirow{2}{*}{ Indicator } & \multicolumn{3}{|c}{ Plaque-forming units per ml } \\
\cline { 2 - 4 } Phage (host) & P15-16 & P28 & M78 \\
\hline PS3 (P15-16) & $3 \times 10^{10}$ & $5 \times 10^{2}$ & $2.5 \times 10^{4}$ \\
PS3 (P28) & $5 \times 10^{3}$ & $6 \times 10^{9}$ & $8 \times 10^{5}$ \\
PS3 (M78) & $4 \times 10^{3}$ & $1 \times 10^{3}$ & $1 \times 10^{9}$ \\
\hline
\end{tabular}

Table 2. Immunological relationship between pyocins and phage PS3.

\begin{tabular}{l|c|c|c|c}
\hline Pyocin or phage & $\begin{array}{c}\text { Pyocin } \mathrm{R} \\
\text { (A) }\end{array}$ & Pyocin R2 & $\begin{array}{c}\text { Phage PS3 } \\
(\mathrm{B})\end{array}$ & A/B \\
\hline Serum & 700 & 700 & 40 & 17 \\
Anti-R & 1,000 & 800 & 0.7 & 1,400 \\
Anti-sheath (R) & 1,400 & 2,000 & 120 & 12 \\
Anti-R2 & 30 & 50 & 400 & $1 / 13$ \\
Anti-PS3 & &
\end{tabular}

patible with the host cannot propagate in the host. Such phenomena are called the modification and restriction. The same situation was found with PS3. The phage was reisolated either on P15-16, P28, or M78 and those phage preparations, PS3 (P15-16), PS3 (P28), and PS3(P78), were assayed with each of the strains. The result is shown in Table 1. It is clearly demonstrated that the efficiency of plating of the phage is high on the last host, while it is very low, $10^{-4}-10^{-8}$, on other strains.

Immunological relationship between the phage and pyocins. As reported in the preceding paper (4), we found four R-type pyocins which were immunologically related. PS3 was also found to be neutralized by the antipyocin $\mathrm{R}$ serum. The antiserum against PS3 was prepared and the reactions between these antisera and the phage or pyocins were investigated quantitatively. Antisera against pyocin R, R2, pyocin R sheath, and PS3 were employed. $\mathrm{K}$ values obtained are summarized in Table 2 . It should be noted that the values in the table can be compared with each other only in a row, not in a column, as the antibody titer of each serum varies with preparations. However, a comparison is meaningful in the last column, the ratio A/B. As already noted in the Materials and Methods, values for pyocins are not too accurate. Taking this point into consideration, pyocins $\mathrm{R}$ and $\mathrm{R} 2$ can be regarded as being immunologically very similar. The main components of these pyocins may be nearly identical. The antiserum against the sheath, the major component of pyocin $\mathrm{R}$, showed a comparable degree of activity to anti-pyocin sera in regard to the neutralization of pyocins. The phageneutralizing ability of anti-pyocin sera was about $1 / 12 \sim 1 / 17$ of those of pyocins, while that of anti-sheath serum was much lower. 
Table 3. Characterization of pyocin- or phage-resistant mutants obtained from strain P15-16.

\begin{tabular}{|c|c|c|c|c|c|c|c|}
\hline $\begin{array}{l}\text { Sensitivity } \\
\text { to } \\
\text { Selected } \\
\text { marker }\end{array}$ & R3 & $\mathrm{R} 4$ & PS3 & $\mathrm{R} 2$ & $\mathrm{R}$ & $\begin{array}{l}\text { Number } \\
\text { scored }\end{array}$ & Type \\
\hline \multirow{3}{*}{ R3r } & $r$ & s & $\mathrm{s}$ & $\mathrm{s}$ & s & 35 & $\mathrm{~b}$ \\
\hline & $r$ & $\mathrm{r}$ & $\mathrm{r}$ & $\mathrm{r}$ & s & 31 & $\mathrm{~d}$ \\
\hline & $r$ & $\mathrm{r}$ & $\mathrm{r}$ & $\mathrm{r}$ & $\mathrm{r}$ & 18 & e \\
\hline \multirow{3}{*}{$\mathrm{R} 4^{\mathrm{r}}$} & $\mathrm{r}$ & $r$ & $\mathrm{r}$ & s & s & 11 & $\mathrm{c}$ \\
\hline & $\mathrm{r}$ & $r$ & $r$ & $\mathrm{r}$ & $\mathrm{s}$ & 39 & $\mathrm{~d}$ \\
\hline & $\mathrm{r}$ & $r$ & $\mathrm{r}$ & $\mathrm{r}$ & $r$ & 17 & e \\
\hline \multirow{3}{*}{$\mathrm{PS}^{\mathrm{r}}$} & $\mathrm{r}$ & $\mathrm{r}$ & $r$ & $\mathrm{~s}$ & s & 42 & $\mathrm{c}$ \\
\hline & $r$ & $\mathrm{r}$ & $r$ & $\mathrm{r}$ & $\mathrm{s}$ & 17 & $\mathrm{~d}$ \\
\hline & $\mathrm{r}$ & $\mathrm{r}$ & $r$ & $\mathrm{r}$ & $r$ & 36 & $\mathrm{e}$ \\
\hline \multirow{2}{*}{$\mathrm{R} 2^{\mathrm{r}}$} & $\mathrm{r}$ & $\mathrm{r}$ & $\mathrm{r}$ & $r$ & $\mathrm{~s}$ & 86 & d \\
\hline & $\mathrm{r}$ & $\mathrm{r}$ & $\mathrm{r}$ & $r$ & $\mathrm{r}$ & 14 & $\mathrm{e}$ \\
\hline \multirow{2}{*}{$\mathrm{Rr}^{\mathrm{r}}$} & $\mathrm{r}$ & $\mathrm{r}$ & $\mathrm{r}$ & $\mathrm{r}$ & $r$ & 48 & $\mathrm{e}$ \\
\hline & $\mathrm{s}$ & s & $\mathrm{s}$ & $\mathrm{s}$ & $r$ & 20 & $\mathrm{f}$ \\
\hline P15-16 & s & s & $\mathrm{s}$ & $\mathrm{s}$ & $\mathrm{s}$ & & $\mathrm{a}$ \\
\hline
\end{tabular}

$\mathrm{s}$ : sensitive $\mathrm{r}$ : resistant, $r$ : selected marker

types $\mathrm{a}-\mathrm{f}$ correspond to those

in Fig. 3.

Reciprocally, anti-phage serum showing a high activity against the phage displayed a moderate inhibition for both pyocins.

Relationship between the phage and pyocins from the aspect of receptor specificity. It is generally accepted that phages as well as bacteriocins exert their biological action through adsorption to their receptors distributed over the cell surface of sensitive bacteria. In $E$. coli it has been known that some colicins share common receptors with certain phages. The receptor substance for pyocin R was prepared by IKEDA and EGAMI (15) from sensitive strains of Pseudomonas aeruginosa and found to be composed of lipopolysaccharide. As we found four kinds of pyocin and a phage which are related immunologically, an experiment was designed to investigate if they have any relation in connection with the receptor. A strain, P15-16, which is sensitive to all the pyocins and to the phage was selected as the starting bacterium. Mutants resistant to each of the pyocins or phage were isolated and their behavior toward other pyocins or the phage was examined. This result is summarized in Table 3. It is clear from the table that the sensitivity toward the phage or pyocins is not independent of each other but correlated in some 


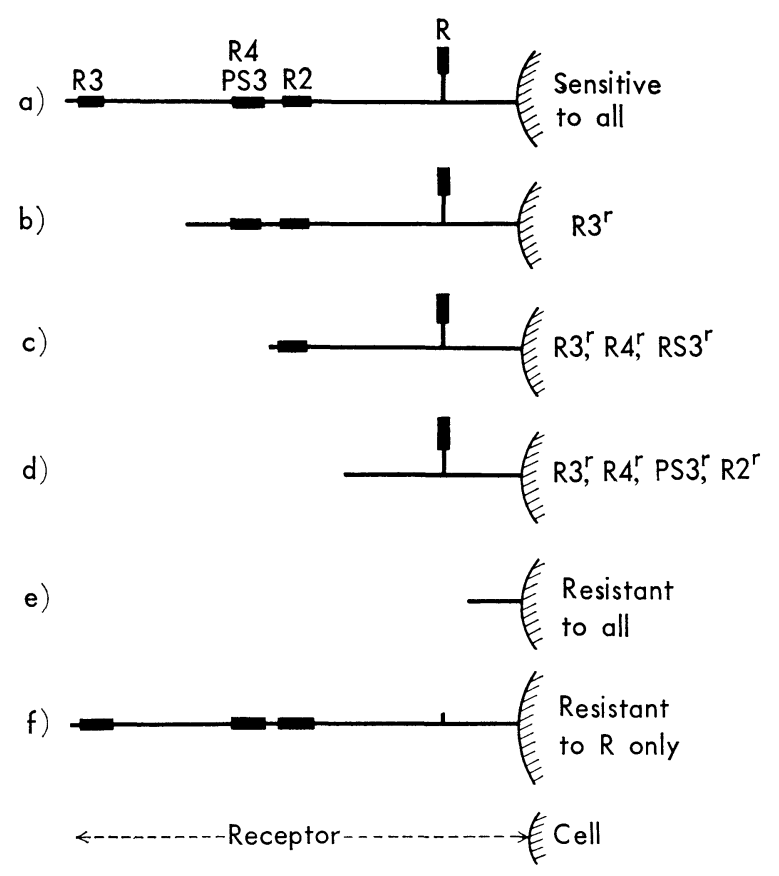

Fig. 3. Schematic representation of the pyocin or phage receptor in the strain P15-16.

Types a) f) correspond to those in Table 3 .

way. General features of the resistance pattern may be summarized as follows: The mutants resistant to pyocin $\mathrm{R}$ were often resistant to all the rest, while mutants resistant to pyocin R2, R3, R4, or PS3 were generally, but not totally, sensitive to pyocin R. Resistance to R2, R4, and PS3 often appeared concomitantly and all the R4-resistant strains so far isolated were resistant to PS3 and vice versa. If the five characters are arranged in a row as listed in the table, any mutant selected as resistant to one of the principle was, in general, also resistant to these placed on the left side of the selected marker.

\section{DISCUSSION}

A number of workers have pointed out the relationship between bacteriophages and bacteriocins, and several speculations have been proposed concerning their origin $(2,3)$. Similarity of the two entities is described at the beginning of this paper. However, there is apparently difficulty in tracing an analogy between the two. Bacteriocins are more like virulent phages in their killing action, while bacteriocinogenic factors resemble lysogenic (tem- 
perate) phages which are generally believed to have no killing action in the tail. Formerly, we found two types of pyocin; R-type which has a structure similar to some phage tails and S-type which is a protein with lower molecular weight than R-types and is not readily resolvable in electron microscopy (4). The present finding of a phage immunologically related to R-type pyocins may shed light on the problem of the origin of bacteriocin at least to some extent.

Morphologically, the tail of the phage PS3 is similar but not identical to R-type pyocins. The phage tail is slightly bigger. The fact that antiserum against pyocin R or R2 could neutralize the phage activity to a considerable extent suggests that there is some common or very similar component(s) in their structure. However the difference in $\mathrm{K}$ values indicates that the relation between the phage and pyocins is not so close as that between pyocins. The antiserum against pyocin sheath showed an effective neutralization for pyocins but very poor or practically none for the phage. This result suggests that the common or closely related component(s) may probably reside in some parts other than the sheath, for instance, core, fiber, or somewhere. This point remains to be solved by the future study. It is not clear whether our phage PS3 is identical to one of the phages isolated by Homma et al. One of them, A $\phi 11$ (11) is morphologically very similar (9).

It is shown that the phage PS3 resembles pyocins not only morphologically and immunologically but also in the receptor specificity. In the wellknown case of colicin $\mathrm{K}$ and phage $\mathrm{T} 6$ which share a common receptor, no immunological relationship was found between them (16).

The result of the analysis of mutants selected as resistant to one of the agents (Table 3) is informative for solving the interrelation of receptor specificity. The structure can be schematically illustrated as in Fig. 3. The absence or presence of a specific site corresponds to the resistance or sensitivity to the agent. Most of the mutants obtained fell into some type of b-f (Table 3) and if the sites are arranged as in Fig. 3, the result can be easily explained without exception. For example, all the mutants resistant to R2 will also be resistant to R3 and R4 as they inevitably lack three sites. Most of $\mathrm{R}^{\mathrm{r}}$ mutants are also resistant to the rest, but some of them remain sensitive to others. This point is explained by assuming a branching out of the $\mathrm{R}$ receptor. The receptor site for PS3 may be identical to that of R4 and must be very close to that of $\mathrm{R} 2$ judging from the frequent appearance of simultaneous resistance.

IKEDA and EGAMI (15) isolated a receptor substance for pyocin $\mathrm{R}$ from a strain which was sensitive to all the agents studied in this paper. The receptor substance was composed mainly of lipopolysaccharide containing glucose, rhamnose, glucosamine, galactosamine, heptose, and ketodeoxyoctonate. Such a kind of chemical study will be very interesting and informative for the elucidation of receptor specificity and the scheme presented in Fig. 3 may be substantialized in that process. 
The discovery of a phage which shares a common or closely related receptor to pyocins offers a convenient tool for the study of action mechanism, origin, and evolution of pyocins. As to the problem of the analogy of bacteriocins to virulent versus temperate phages commented before, it seems to us that the difference between virulent and temperate is not strict but conditional. The phage PS3 behaves just like a virulent phage in the strains P15-16, P14, and M78 but like a typical temperate phage in some other strains.

The authors are indebted to Miss Chie Yui for the gift of antiserum against pyocin $\mathrm{R}$ sheath. Collaboration of Miss Michiko Hiramaru in the work of receptor analysis is also gratefully acknowledged. The authors express their thanks to Miss A. Watanuki, Mr. H. Yamagata, and Dr. H. Uchida, Institute of Medical Science, for the preparation of antisera, and to Mr. Y. Futaesaku, Tokyo Medical and Dental University, for the electron microscopy. Thanks are also due to Prof. F. Egami for his advice and encouragement throughout this study. The expense of this study was defrayed in part by the Grant-in-Aid for Scientific Research from the Ministry of Education.

\section{REFERENCES}

1) P. FredericQ, Symp. Soc. Exptl. Biol., 12, 104 (1958).

2) P. Fredericq, J. Theoret. Biol., 4, 159 (1963).

3) D.E. BRadley, Bacteriol. Rev., 31, 230 (1967).

4) S. Ito, M. Kageyama and F. Egami, J. Gen. Appl. Microbiol., 16, 31 (1970).

5) H. Endo, K. Ayabe, K. Amako and K. Takeya, Virology, 25, 469 (1964).

6) D.E. Bradley and C.A. Dewar, J. Gen. Microbiol., 45, 399 (1966).

7) K. Okamoto, J.A. Mudd, J. Mangan, W.M. Huang, J.V. Subbaiah and J. Marmur, J. Mol. Biol., 34, 413 (1968).

8) M. HaAs and H. Yoshikawa, J. Virology, 3, 233 (1969).

9) J.Y. Homma, H. Watabe and Y. Tanabe, Japan. J. Exptl. Med., 38, 213 (1968).

10) J.Y. Homma and H. ShionoyA, Japan. J. Exptl. Med., 37, 395 (1967).

11) M.H. AdAmS, Bacteriophages, Interscience Publishers, Inc., New York (1959).

12) M. Kageyama and F. Egami, Life Sciences, 471 (1962).

13) M. Kageyama, J. Biochem. (Tokyo), 55, 49 (1964).

14) C. Yui, F. EgAmi and S. IsHiI, Seikagaku, 41, 439 (1969).

15) K. Ikeda and F. Egami, J. Biochem. (Tokyo), 65, 603 (1969).

16) W.F. Goebel, G.T. BArry, M.i. Jesaitis and E.M. Miller, Nature, 176, 700 (1955).

17) S. Ishiı, Y. Nishi and F. Egami, J. Mol. Biol., 13, 428 (1965). 